\title{
$\mid$\begin{tabular}{|l} 
Sustainable Business \\
International Journal
\end{tabular}
}

NÚMERO 11 - JANEIRO DE 2012

ISSN 1807-5908

\section{ANÁLISE DO CONHECIMENTO COM O USO DAS REDES SOCIAIS}

Tatiana Barbosa de Azevedo, MSc.

Professora do CEFET-MG

E-mail: t4tiana@yahoo.com.br

Martius Vicente Rodriguez Y Rodriguez, Dr.

Professor do Curso de Mestrado em Sistemas de Gestão - LATEC/UFF

E-mail: martiusyrodriguez@gmail.com

\section{RESUMO}

Estamos vivenciando a era do conhecimento. O capital intelectual das organizações, dos mais variados setores, jamais foi tão valorizado. Atualmente, a detenção do conhecimento está diretamente ligada ao sucesso das organizações.

Neste cenário, destacam-se as redes sociais, onde indivíduos e organizações se relacionam focados na potencialização do conhecimento. Estas redes servem como um instrumento para aquisição, troca e disseminação de conhecimento entre seus colaboradores, na busca de melhoria de desempenho e eficiência.

Neste contexto, o entendimento dos relacionamentos informais nos ambientes inter e intraorganizacionais, torna-se um campo de estudo que vem ganhando grande amplitude. Este entendimento torna-se possível através da utilização de ferramentas de análise de redes sociais. Através destas ferramentas, é possível mapear as relações entre os indivíduos, instituições e organizações.

Visualizando esta realidade, este artigo pretende descrever os principais conceitos relacionados à criação do conhecimento através da análise de redes sociais.

Palavras-chave: Gestão do Conhecimento, Redes Sociais, Análise de Redes Sociais. 


\begin{abstract}
We are experiencing the knowledge age. The intellectual capital of organizations in various sectors, has never been so valued. Possession of knowledge is directly linked to the success of organizations.

In this scenario, there are the social networks, where individuals and related organizations, are focused on the enhancement of knowledge. These networks serve as a tool for acquisition, exchange and dissemination of knowledge among their members, in the search to improve performance and efficiency.

In this context, the understanding of informal relationships in inter-and intraorganizational settings, becomes a field of study that is gaining importance. This understanding is made possible through the use of tools of social network analysis. Through these tools, you can map the relationships between individuals, institutions and organizations. In view of this, this article aims to describe the main concepts related to knowledge creation in the informal relationships, through social network analysis.
\end{abstract}

Keywords: Knowledge Management, Social Networks, Social Network Analysis. 


\section{INTRODUÇÃO}

Diante da globalização e grande competitividade, a detenção do conhecimento está diretamente relacionada ao desempenho de um indivíduo ou de uma organização.

Torna-se claro aos gestores de mentes diferenciadas, que a gestão do conhecimento é ponto central para que um indivíduo esteja à frente de seus competidores e preparado para uma realidade de mercado com mudanças aceleradas e de múltiplas dimensões.

Nesta perspectiva de valorização do conhecimento, destacam-se as redes sociais informais, que vislumbram uma reunião de indivíduos de diferentes competências, especialidades, características e organizações, em busca de potencializar a fluência e consistência do conhecimento.

Atualmente várias destas redes estão sendo criadas e fomentadas por diferentes indivíduos e organizações, com foco na aquisição, troca, criação e disseminação de conhecimento entre seus colaboradores, na busca de melhoria de desempenho e eficiência para o enfrentamento das mudanças inevitáveis, tornando possível sobreviver neste ambiente competitivo.

Para possibilitar o entendimento destas redes informais, é necessário analisar suas características e peculiaridades. Além de "controlar" estas relações, às voltas com o desafio de melhor gerir a troca de conhecimento entre seus colaboradores e se colocar em posição de vantagem no mercado.

Neste cenário, de valorização do capital intelectual, se enquadra este artigo. Que tem como objetivo principal descrever como a utilização de ferramentas estratégicas de análise de redes sociais, pode auxiliar na análise do fluxo de informações e das conexões das redes sociais informais, e potencializar o processo decisório e a ampliação do conhecimento dos indivíduos a ela interligados.

\subsection{Problema}

Segundo Nonaka (1985), para uma eficiente gestão do conhecimento, é de suma importância atentar aos relacionamentos informais das redes sociais inter e intraorganizacionais.

Este é o problema a ser tratado por este artigo: analisar a criação de conhecimento nas redes sociais informais, através da aplicação de ferramenta de análise de redes sociais. 


\section{2 - Metodologia}

Como forma de tecer os argumentos necessários para sustentar esta afirmação foi feita pesquisa bibliográfica, na busca de uma melhor compreensão da utilização das ferramentas de análise de redes sociais em prol de uma melhoria da gestão do conhecimento.

Quanto ao meio de investigação bibliográfico, foram realizados estudos em materiais científicos, dados de congressos, artigos, revistas científicas, publicações, internet e bibliotecas, em busca de conceitos inovadores sobre gestão do conhecimento, redes sociais e análise de redes sociais, combinados a estes uma análise histórica dos estudos de redes que se destacam nos últimos anos.

\section{GESTÃO DO CONHECIMENTO}

Muito se fala sobre as origens da gestão do conhecimento, fala-se que as primeiras tentativas de gerenciar o conhecimento surgiram com os egípcios e gregos na era de Platão. Não se pode negar esta possível origem, além da grande influência de Taylor, com a primeira evidência de codificação do conhecimento.

Apesar das contribuições de todos, não se pode afirmar que a gestão do conhecimento, como movimento, tenha iniciado em qualquer um destes momentos. As primeiras iniciativas organizacionais começaram no fim da década de 80 , em sua maioria com um enfoque tecnológico.

A primeira grande onda chegou com a publicação do clássico trabalho de Nonaka e Takeuchi em 1995, The Knowledge Creating Company, sobre o processo de criação de conhecimento organizacional como fonte de inovação, explicando o grande sucesso das empresas japonesas sobre as americanas. "Uma empresa criadora de conhecimento não opera em um sistema fechado, mas em um sistema aberto, no qual existe um intercâmbio constante de conhecimento com o ambiente externo." (Nonaka, 1995).

A gestão do conhecimento é um processo rico e complexo de criação e disseminação contínua de conhecimento de produtos, processos, tecnologias, etc..

$\mathrm{Na}$ atualidade, o principal valor das organizações, não está apenas em seu capital físico, mas sim, em seu conjunto de talentos, idéias, capacidades, enfim, em seu capital intelectual. "Nenhum investidor compra ações da Microsoft ou da Intel em virtude das fábricas e 
equipamentos que possuem, mas sim por suas capacidades de gerarem novas idéias, habilidades e inovações capazes de gerar riqueza." (Stewart, 1998, p.9).

Neste novo cenário de importância ao conhecimento no contexto organizacional e econômico, e da valorização do capital intelectual, o gerenciamento deste conhecimento dentro das organizações torna-se fundamental para o melhor posicionamento da empresa no mercado atual.

A criação e utilização do conhecimento são feitas principalmente através da conversão de conhecimento tácito em conhecimento explícito, e vice-versa. Este ciclo tornou-se conhecido na literatura como modelo SECI, ou espiral SECI. Segundo este modelo retrata a criação do conhecimento inicia com a socialização e passa pela conversão deste conhecimento em forma de espiral. Em cada modo de conversão, o conhecimento é amplificado e podem ser descritos desta maneira:

1 - Socialização: consiste em compartilhar e criar conhecimento tácito de indivíduo para indivíduo.

2 - Externalização: consiste em articular conhecimento tácito através do diálogo e reflexão.

3 - Combinação: consiste em sistematizar e aplicar o conhecimento explícito e a informação.

4 - Internalização: consiste em aprender e adquirir novo conhecimento tácito na prática. 


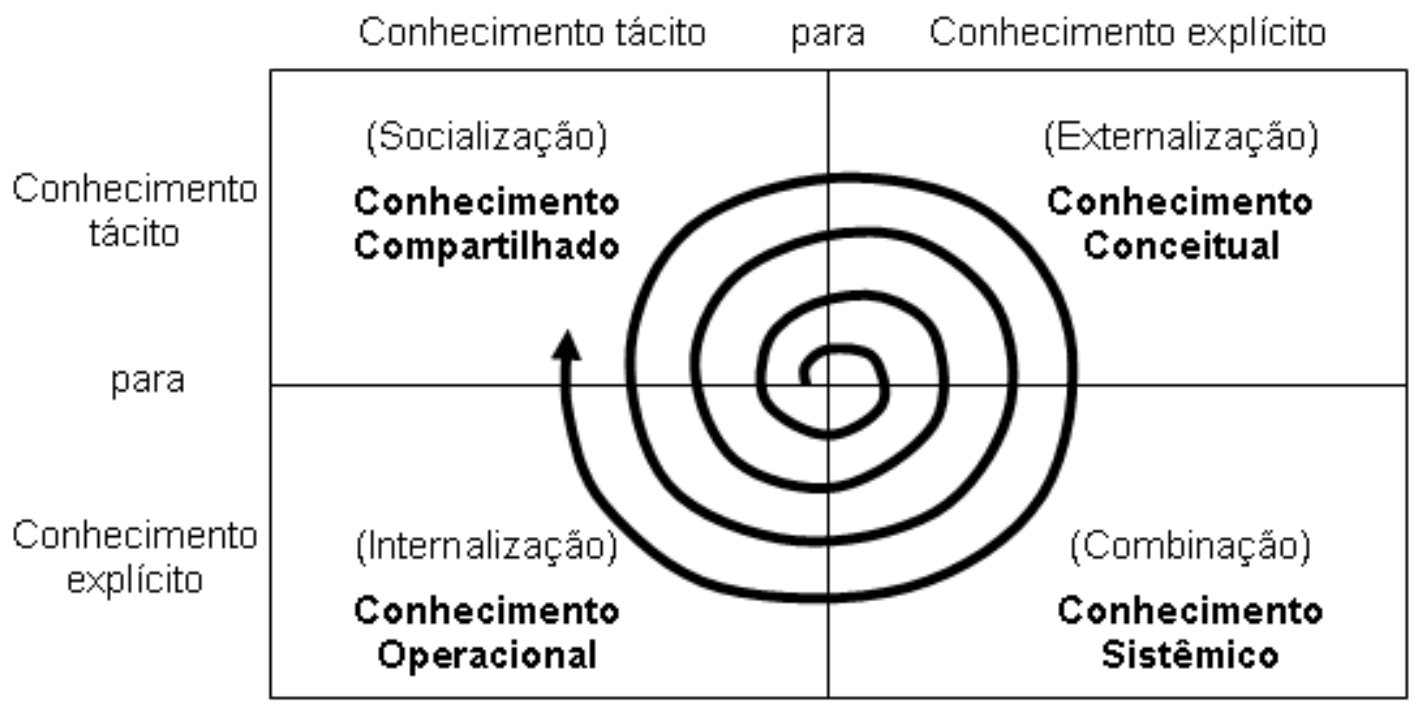

Figura 01 - Espiral do Conhecimento - Modelo SECI.

Fonte: Nonaka e Takeuchi (2008).

Para que este processo de criação e disseminação do conhecimento ocorra, é necessário prover aos indivíduos envolvidos no processo, um ambiente com liberdade, segurança, em um espaço que encoraje todos os indivíduos envolvidos a engajar-se no processo espiralado da criação do conhecimento. Este local, ou contexto físico, de criação do conhecimento é conhecido como ba. Segundo Bohm (1996) ba é o tempo e o espaço fenomenológico onde o conhecimento emerge, como uma "corrente de significado".

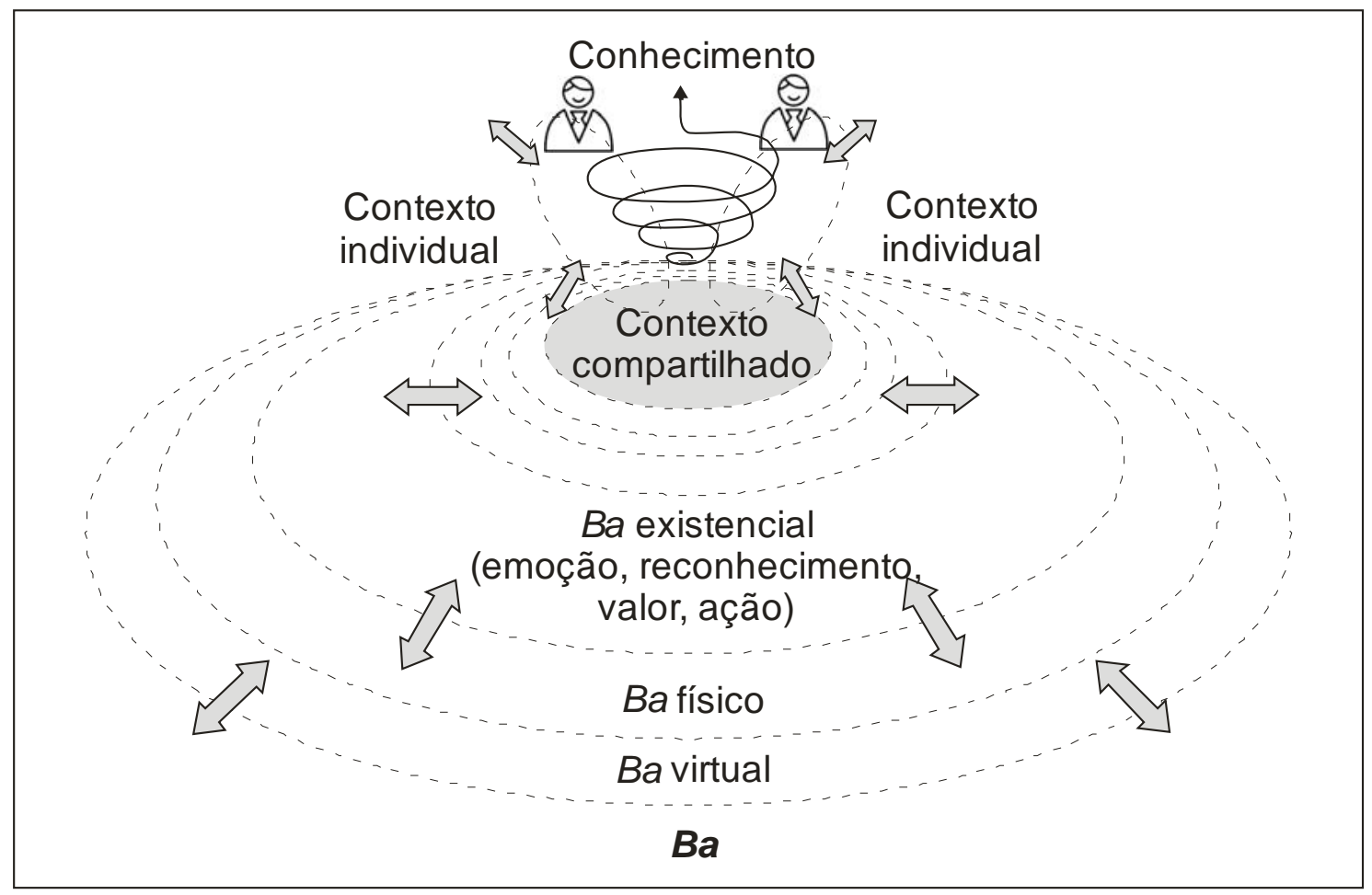


Figura 02 - Representação Conceitual do ba.

Fonte: Nonaka e Takeuchi (2000).

Este cenário propício para troca de conhecimento é encontrado nas redes organizacionais e interorganizacionais, onde os indivíduos conectados encontram um ambiente horizontalizado, seguro, livre no qual todos os atores (nós da rede) estão conectados com o mesmo propósito: ampliar seus conhecimentos.

Segundo Nonaka e Takeuchi (1995, p.59): "A criação do conhecimento organizacional, portanto, deve ser entendida como um processo que amplifica 'organizacionalmente' o conhecimento criado por indivíduos e cristaliza-o como parte da rede de conhecimentos da organização. Esse processo ocorre dentro de uma 'comunidade de interação' em expansão, que atravessa os níveis das fronteiras intra e interorganizacionais.”.

\section{REDES - UMA DEFINIÇÃO}

O estudo das redes possui vários enfoques. Os primeiros estudos partiram das ciências exatas, através da criação da teoria dos grafos. O grafo é um conjunto de nós, conectados por arestas, que em conjunto, formam uma rede. Mais tarde os estudos passaram pelas áreas das ciências biológicas, depois pela sociologia e antropologia, momento em que assume a definição de formas específicas de interação entre indivíduos e grupos familiares, grupos sociais, grupos de trabalho, etc..

Os autores enfatizam a constante mutação e a complexidade que o termo rede sofre dependendo do contexto em que é utilizado, podendo este, assumir diferentes sentidos conforme a área de conhecimento.

Seguindo a visão de Castells (2003) podemos de forma simplificada, considerar que: Rede é um conjunto de nós interconectados. Nó é o ponto no qual uma curva se entrecorta.

Trata-se de uma definição básica, mas que define a forma estrutural de uma rede de elementos interligados. 


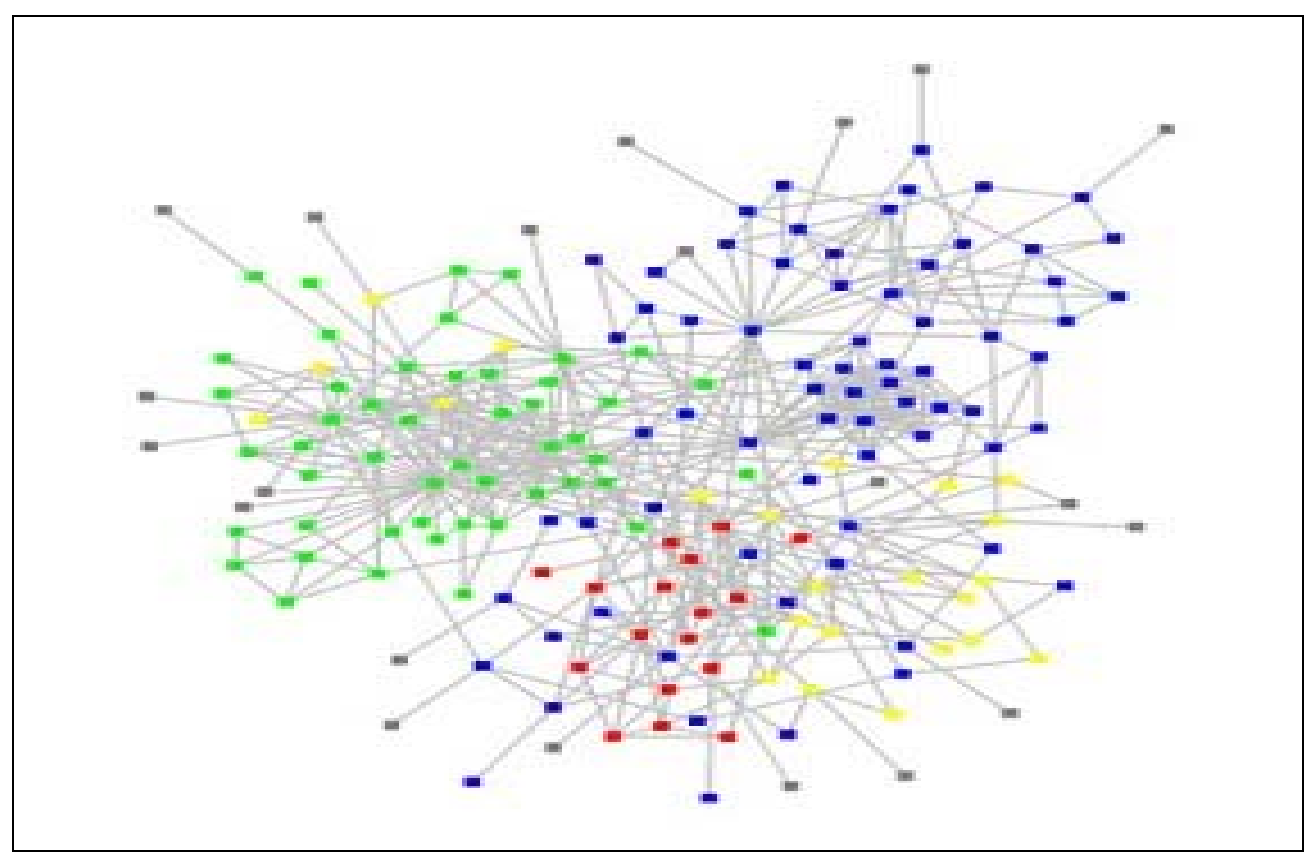

Figura 03 - Representação de uma rede de elementos interligados.

Fonte: Autor

Num sentido etimológico o termo "rede" é derivado do latim (retis), e significa "entrelaçamento de fios, cordas, cordéis, arames com aberturas regulares fixadas por malhas, formando uma espécie de tecido". A partir da noção de estrutura reticulada e entrelaçamento a palavra foi ganhando novos significados ao longo do tempo e abordagens cada vez mais interdisciplinares. (Loiola; Moura, 1997).

Uma rede é fundada na multiplicidade de seus elementos participantes e principalmente na horizontalidade das interligações. A rede é um tipo de organização específico, que se distingue das demais por conta de seus aspectos formais e seus aspectos processuais ou operacionais.

Dentro da bibliografia existem inúmeras descrições para os termos rede, tais como:

- “uma rede, por definição, não tem centro, mas apenas nós de diferentes dimensões e relações internodais que são freqüentemente assimétricas. Entretanto, em última instância, todos os nós são necessários para a existência da rede” (Castells, 1998,p.11);

- "o termo rede, em sua multiplicidade, nos remete tanto a uma dimensão conceitual como a uma vertente instrumental". Rede é uma proposta de ação, "um modo espontâneo de organização em oposição a uma dimensão formal e instituída" (Junqueira, 1998, p. 96); 
A teoria das redes vem sendo estudada e analisada por vários autores de diferentes áreas e visões, conforme quadro de temas de redes estudados ao longo dos últimos anos:

\begin{tabular}{|c|c|c|}
\hline \multirow[t]{5}{*}{$\begin{array}{l}\text { Década de } \\
50 \text { e } 60\end{array}$} & $\begin{array}{l}\text { Bavelas (1950); Bavelas e Barret } \\
\text { (1951); Leavitt }\end{array}$ & Resolução de problema em grupo \\
\hline & Coleman, Katz e Menzel (1957) & Difusão e adoção de inovações \\
\hline & Kadushin (1966) & Suporte social \\
\hline & Michell (1969) & Fenômenos urbanos complexos \\
\hline & Kapferer (1969) & Formação de coalisão grupal \\
\hline \multirow[t]{4}{*}{$\begin{array}{l}\text { Década de } \\
70\end{array}$} & $\begin{array}{l}\text { Laumann e Pappi (1973); Laumann, } \\
\text { Marsden e Galaskievwics (1977); } \\
\text { Wellman (1979) }\end{array}$ & Tomada de decisão em comunidade \\
\hline & Cook (1977) & Poder e redes interorganizacionais \\
\hline & Zachary (1977) & Formação de coalizão \\
\hline & Rogers (1979) & Difusão e adoção de inovações \\
\hline \multirow[t]{7}{*}{$\begin{array}{l}\text { Década de } \\
\qquad 80\end{array}$} & Thurman (1980) & Formação de coalizão \\
\hline & Fischer (1982) & Urbanização e bem estar individual \\
\hline & Brass (1985) & $\begin{array}{l}\text { Influencias de gênero nas relações } \\
\text { intraorganizacionais }\end{array}$ \\
\hline & Krackhardt e PORter (1986) & $\begin{array}{l}\text { Influências individuais na } \\
\text { rotatividade }\end{array}$ \\
\hline & $\begin{array}{l}\text { Krackhardt (1987); Freeman, } \\
\text { Romney, e Reeman } 1987\end{array}$ & Cognição e percepção social \\
\hline & $\begin{array}{l}\text { Krackhardt e Stern (1988); Nelson } \\
\text { (1989) }\end{array}$ & $\begin{array}{l}\text { Resposta a crise e conflito nas } \\
\text { organizações }\end{array}$ \\
\hline & Krackhardt (1987), 1988 e 1992 & $\begin{array}{l}\text { Procedimentos QAP e modelos } \\
\text { estatísticos }\end{array}$ \\
\hline
\end{tabular}




\begin{tabular}{|c|c|c|}
\hline $\begin{array}{l}\text { Década de } \\
90\end{array}$ & Krackhardt e Kilduff (1990) & $\begin{array}{l}\text { Cultura organizacional e relações } \\
\text { informais de amizade }\end{array}$ \\
\hline & $\begin{array}{l}\text { Krackhardt (1990); Ibarra e Andrews } \\
\text { (1995); Krackhardt e Carley (1998) }\end{array}$ & $\begin{array}{l}\text { Relações de poder, sensemaking e } \\
\text { impacto em inovações }\end{array}$ \\
\hline & Krackhardt e Kilduff (1994) & Reputação \\
\hline & $\begin{array}{l}\text { Tolbert, Salancik, Krackhardt e } \\
\text { Andrews (1995), Krackhardt e Carley } \\
\text { (1998) }\end{array}$ & $\begin{array}{l}\text { Efeitos individuais de posições na } \\
\text { rede }\end{array}$ \\
\hline & Podolny, Stuart e Hannan (1996) & Redes interorganizacionais \\
\hline & $\begin{array}{l}\text { Doreian , Kapuscincski, Krackhardt e } \\
\text { Szczypula (1996) }\end{array}$ & $\begin{array}{l}\text { Transitividade, reciprocidade e } \\
\text { equilíbrio grupal }\end{array}$ \\
\hline & Wasserman e Pattison (1996) & Modelos estatísticos \\
\hline & $\begin{array}{l}\text { Ibarra (1993); Smith-Lovin e Ibarra } \\
\text { (1997); Mehra, Kilduff e Brass (1998) }\end{array}$ & Gênero e redes intraorganizacionais \\
\hline & Labianca, Brass e Gray (1998) & Percepção de conflito intergrupal \\
\hline & Brass, Butterfield e Skaggs (1998) & $\begin{array}{l}\text { Relações e comportamento anti- } \\
\text { ético }\end{array}$ \\
\hline & Kuipers (1999) & Tipologia de redes informais \\
\hline & Castells (1999) & A sociedade em rede \\
\hline $\begin{array}{l}\text { A partir do } \\
\text { ano } 2000\end{array}$ & $\begin{array}{l}\text { Carvalho (2000); Candido e Abreu } \\
\text { (2000), Oliver (2001), Hasegawa e } \\
\text { Furtado (2001) e Penno (2002) }\end{array}$ & Redes interorganizacionais \\
\hline & Mehra, Kilduff e Brass (2001) & Redes de alto e baixo desempenho \\
\hline & Lazer (2001) & Rede e atitude individual \\
\hline & $\begin{array}{l}\text { Nascimento (2000), Marteleto (2001), } \\
\text { Lai e Wong (2002) }\end{array}$ & $\begin{array}{l}\text { Transferência de informações em } \\
\text { redes }\end{array}$ \\
\hline & Sluzki (1997 e 200) & $\begin{array}{l}\text { Alternativa terapêutica e saúde } \\
\text { mental }\end{array}$ \\
\hline
\end{tabular}




\begin{tabular}{|l|l|l|}
\hline & Najmanovich (2002) & Novo paradigma e rede \\
\hline & Kadushin & Motivação \\
\hline & $\begin{array}{l}\text { Ceenders (2002) } \\
\text { Borgatti e Parker (2002) }\end{array}$ & Modelo matemático \\
\hline & Fernandez (2002) & Redes informais \\
\hline & Tsai e Kilduff (2003) & $\begin{array}{l}\text { Intervenção em comunidades } \\
\text { intraorganizacional }\end{array}$ \\
\hline
\end{tabular}

Quadro 01 - Temas sobre redes sociais estudados.

Fonte: Wasserman e Faust (1999, p.6).

Segundo Martinho (2003), a articulação em rede passou a ser valorizada no final da década de 80 e início da década de 90, especialmente devido ao uso das redes eletrônicas. A partir dessa época, a função da rede foi explicitada e reconhecida e começou a ser considerada um elemento político na sustentação dos direitos sociais da nação. 


\section{REDES SOCIAIS}

Uma rede social refere-se a um conjunto de pessoas (organizações ou entidades) conectadas por relacionamentos sociais, motivadas pela amizade, relação de trabalho ou troca de informação - uma representação formal de atores e suas relações. O fenômeno da conectividade é que constitui a dinâmica das redes e existe apenas na medida em que as conexões forem estabelecidas.(Marteleto, 2001)

Segundo J.Scott (2000), três vertentes embasaram a atual teoria de redes sociais:

1 - Analistas sociométricos - estudo de pequenos grupos tendo os grafos como ferramenta- que, nos anos 1930, trabalharam em pequenos grupos e produziram muitos avanços técnicos com métodos da teoria dos grafos; tradição gestaltiana, da psicologia, com destaque para os autores Kurt Lewin, Jacob Moreno, Fritz Heider;

2 - Pesquisadores de Harvard que, também nos anos 1930, exploraram padrões de relações interpessoais informais e a formação de "cliques", blocks, "clusters"; autores influenciados por Radcliffe-Browun e Durkheim;

3 - Antropólogos de Manchester, que usaram os conceitos das duas primeiras vertentes para investigar a estrutura de relações comunitárias em sociedades tribais e pequenas vilas. São representantes John Barnes e Clyde Mitchell, também influenciados por Radcliffe-Brown, enfatizando a integração e coesão, o conflito e a mudança. Essas três correntes foram reunidas novamente em Harvard nas décadas de 1960 e 1970, quando se forjaram as bases da moderna teoria de análise de redes sociais. 


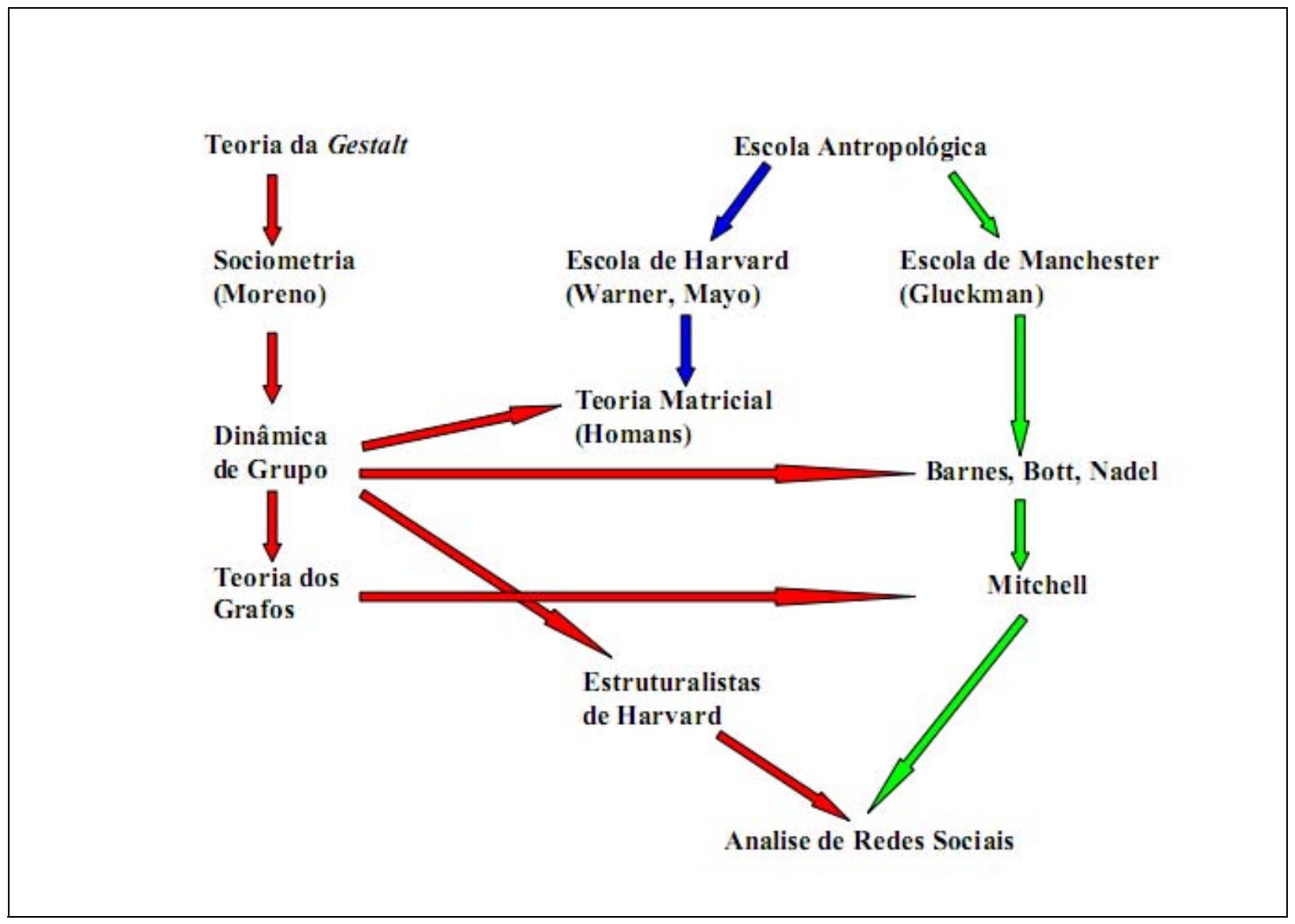

Figura 04 - Origem da Análise de Redes Sociais.

Fonte: Scott (2000 p.8).

Ao analisar a estrutura das redes em uma organização, deve-se ter em mente que as redes são formadas por múltiplas relações internas e externas. Nesta perspectiva, e dentro do contexto da teoria organizacional, podemos analisar as redes nos aspectos intra e interorganizacionais. No aspecto intraorganizacional, também conhecido por microambiente, estarão sendo analisados apenas os aspectos internos da organização, tais como relação entre as pessoas, os setores, o processo produtivo, etc..

No aspecto interoganizacional, estarão sendo analisadas as relações externas à organização. Estas ligações compreendem laços pessoais, alianças estratégicas com fornecedores, clientes, transações comerciais, fluxos de recursos, fluxos de informações, dentre outras. O objeto do estudo das redes é analisar estas estruturas, seus impactos e evolução. 


\section{ANÁLISE DE REDES SOCIAIS}

A análise de redes é uma forma de análise de dados que difere dos métodos tradicionais de análise. Ela tem sido utilizada nas análises sociais, subsidiando pesquisadores para descrever fenômenos empíricos onde se dá importância às interações entre os atores de um determinado contexto social.

Em uma rede, os relacionamentos podem ser de diversos tipos: econômicos, políticos, afetivos e sociais.

A noção do que se chama redes sociais e os métodos de análise dessas redes têm sido bastante usado na comunidade científica para analisar relacionamentos entre entidades sociais e os padrões e implicações desses relacionamentos. (Wasserman e Faust,1994)

Segundo Martelo (2005), a análise de redes estabelece um novo paradigma na pesquisa sobre a estrutura social. Para estudar como os comportamentos ou as opiniões dos indivíduos dependem das estruturas nas quais eles se inserem, a unidade de análise não são os atributos individuais (classe, sexo, idade, gênero), mas o conjunto de relações que os indivíduos estabelecem através das suas interações uns com os outros.

A estrutura é definida também como uma rede de relações e de limitações que exerce influência nas escolhas, nas orientações, nos comportamentos, e opiniões dos indivíduos.

Os principais conceitos que embasam a análise de redes sociais são:

- Ator: é considerado ator qualquer entidade existente no contexto da rede que participe ou não dos processos de inovação podendo ser uma unidade coletiva, corporativa ou individual.;

- Vínculo relacional: é uma ligação mantida entre atores;

- Relação: é uma coleção de vínculos relacionais de um tipo específico entre atores de um grupo;

- Subgrupo: é um subconjunto de atores e todos os vínculos relacionais entre eles;

- Rede social: consiste de um conjunto finito de atores e as relações existentes entre eles.

Segundo Soares (2002), a análise de redes compreende os seguintes princípios:

1 - os atores e suas ações são vistos como interdependentes, em vez de unidades autônomas; 
2 - os vínculos relacionais entre atores são canais de transferência ou fluxo de recursos (materiais ou não-materiais);

3 - os modelos de rede que centram atenção sobre os indivíduos vêem o ambiente estrutural da rede como algo que pode fornecer oportunidades ou constrangimentos à ação individual;

4 - os modelos de rede conceituam estrutura (social, econômica, política e assim por diante) como padrões duradouros de relações entre atores;

5 - a estrutura afeta formalmente a ação, por meio de um determinismo fraco estruturas altamente homogêneas induzem certas práticas, por causa do tipo de contatos que elas favorecem;

6 - a estrutura afeta a percepção do próprio interesse - por exemplo, qualquer pessoa que quer casar perceberá um cônjuge elegível do próprio status social, como a escolha mais econômica;

7 - os indivíduos racionais tomam decisões de acordo com próprio interesse, isto é, com base numa escala de preferências - racionalidade relativa;

O autor destaca também, a importância de reconhecer que a unidade de análise, no campo das redes sociais, não é o ator individual, mas uma entidade, que consiste no conjunto de atores e nas ligações/laços entre eles.

Segundo Marteleto (2001), a rede social representa um conjunto de participantes autônomos, unindo idéias e recursos em torno de valores e interesses compartilhados. As relações podem ser direcionais ou não direcionais e as redes podem ter mais de uma relação. Um par de atores que formam uma relação denomina-se uma díade. Para cada conjunto de díades tem-se um grafo. O objetivo da análise de redes sociais é demonstrar que a análise de uma díade só tem sentido em relação ao conjunto das outras díades da rede, porque sua posição estrutural tem necessariamente um efeito sobre sua forma, seu conteúdo e sua função.

A análise de redes sociais não toma como unidade de análise o ator individual que faz parte da rede em estudo, mas a coleção de atores ou indivíduos e as suas interações.(Wasserman e Faust ,1994). 


\section{CONSIDERAÇÕES FINAIS}

Observa-se que a fundamentação teórica que respalda o estudo sobre redes sociais está diretamente ligada com a gestão do conhecimento.

Com o crescimento do número de indivíduos e organizações interligados em redes, tornou-se necessário aprimorar a análise e entendimento destas redes, cujas conexões geram um grande fluxo de informações entre indivíduos com propostas e interesses similares, no âmbito pessoal ou profissional.

Para isto, utiliza-se a análise de redes sociais, uma ferramenta estratégica, capaz de auxiliar no entendimento e controle do processo de criação, troca e disseminação do conhecimento nas das redes informais, além de identificar atores fundamentais em uma organização.

Esta análise é realizada através do estudo dos fluxos das informações, das formas de conexões estabelecidas, dos atores envolvidos em uma determinada rede social. É uma ferramenta de análise de dados importante, com propósitos que vão desde o diagnóstico de situações reais até a predição de comportamentos futuros de um grupo de indivíduos ou em uma organização. A idéia central deste artigo foi a de propor uma breve discussão teórica a respeito de como é possível potencializar a criação e disseminação do conhecimento através da utilização das ferramentas de análise de redes sociais.

Assim, espera-se que o leitor entenda que a compreensão do processo de troca de informação em redes sociais pode ser uma boa estratégia para a melhor gestão do conhecimento. 


\section{REFERÊNCIAS BIBLIOGRÁFICAS}

BOHM, David. On Dialogue. Londres: Routledge, 1996.

CASTELLS, Manuel. La era de la informacion: economia, sociedade y cultura. Madrid: Alianza Editorial, 1998.

CASTELLS, Manuel. A sociedade em rede. São Paulo: Paz e Terra, 2003.

FLEURY, Maria Terez Lima e OLIVEIRA JUNIOR, Moacir de Miranda. Gestão Estratégica do Conhecimento: Integrando Aprendizado, conhecimento e competências. São Paulo: Atlas, 2001.

GUEDES, André Luis Azevedo. "A ocorrência da co-geração do conhecimento no desenvolvimento de produtos e serviços”. UFF, Niterói, 2008.

JUNQUEIRA, Luciano. Descentralización, y red en la gestión de la ciudad. Reforma y Democracia. Venezuela: Revista del CLAD, 1998.

KAPLAN, Robert e Norton, David. Estratégia em Ação. Rio de Janeiro: Editora Campus, 1998.

LIMA, Karina K., AMARAL, Daniel. "Práticas de gestão do conhecimento em grupos de pesquisa da rede Instituto Fábrica Milênio”. Gest. Prod., São Carlos, 2008.

LOIOLA, E.; MOURA, S. Análise de redes: uma contribuição aos estudos organizacionais. In: FISCHER, T. (Org.). Gestão Contemporânea: cidades estratégicas e organizações locais. Rio de Janeiro: Fundação Getúlio Vargas, 1997.

MARTELETO, Regina Maria . Análise de redes sociais : aplicação nos estudos de transferência da informação. Ciência da Informação. Brasília, v.30, p. 71-81, Instituto Brasileiro de Informação em Ciência e Tecnologia. 2001

MARTELO. "Análise de Redes Sociais - Aplicação nos Estudos de Transferência da Informação" - Programa de Pós-Graduação em Ciência da Informação, MCT/IBICT $\mathrm{UFRJ} / \mathrm{ECO}, 2001$

MARTINHO, C. Redes: uma introdução às dinâmicas da conectividade e da autoorganização. Brasília: WWF, 2003. 
MENEGHELLI, Regina Buzetti. "Redes sociais informais nas organizações e satisfação no trabalho: estudo de caso numa empresa de energia”. UFF, Niterói, 2009.

MONTEIRO, Samuel, CARDOSO, Leonor. "Impacto(s) da gestão de recursos humanos na gestão do conhecimento em organizações industriais". Comportamento Organizacional e Gestão, 2008.

NONAKA, Ikujiro.; TAKEUCHI, Hirotaka. Criação de conhecimento na empresa: como as empresas Japonesas geram a dinâmica da inovação. Rio de Janeiro: Elsevier, 1995.

RODRIGUEZ, Martius V. Rodriguez. Gestão Empresarial em Organizações Aprendizes. Rio de Janeiro: QualityMark Editora, 2006.

ROSSETI, Adroaldo Guimarães, MORALES, Aran B.T.. "O papel da tecnologia da informação na gestão do conhecimento”. Ci. Inf., Brasília, v.36, n.1, p. 124-135, 2007

SCOTT, J. Social Network analysis. Califórnia: Sage Publications, 2000.

SENGE, Peter, M.. A Quinta Disciplina. São Paulo: Best Seller, 1998.

SILVA, Sergio, ROZENFELD, Henrique. "Proposição de um modelo para avaliar a gestão do conhecimento no processo de desenvolvimento de produtos”. Ci. Inf., Brasília, v.36, n.1, p. 147-157, 2007.

SOARES, Weber. "Da metáfora à substância: redes sociais, redes migratórias e migração nacional e internacional em Valadares e Ipatinga.". Tese de Doutorado, UFMG/Cedeplar, Belo Horizonte, 2002.

STEWART, T. A. Capital Intelectual. Rio de Janeiro: Campus, 19.

SVEIBY, Karl Eril. A nova riqueza das organizações: gerenciando e avaliando patrimônios de conhecimento. Rio de Janeiro: Campus, 1998.

TAKEUCHI, Hirotaka e NONAKA Ikujiro. Gestão do Conhecimento. Porto Alegre: Bookman, 2008.

TAKEUCHI, Hirotaka e NONAKA Ikujiro. Criação do Conhecimento na Empresa. Rio de Janeiro: Campus, 1997. 
WASSERMAN, Stanley.; GALASKIEWICZ, Joseph. Advances in social network analysis: research In the social and behavioral sciences. Thousand Oaks, Calif.: Sage Publications, 1994. 